\title{
Neighbourhood immigrant acculturation and diet among Hispanic female residents of New York City
}

\author{
Yoosun Park ${ }^{1}$, Kathryn Neckerman ${ }^{2}$, James Quinn ${ }^{3}$, Christopher Weiss ${ }^{3}$, \\ Judith Jacobson ${ }^{4}$ and Andrew Rundle ${ }^{4, *}$ \\ ${ }^{1}$ School for Social Work, Smith College, Northampton, MA, USA: ${ }^{2}$ The Center for Health and Social Sciences, \\ The University of Chicago, Chicago, IL, USA: ${ }^{3}$ Institute for Social and Economic Research Policy, Columbia \\ University, New York, NY, USA: ${ }^{4}$ Department of Epidemiology, Mailman School of Public Health, Columbia \\ University, 722 West 168th Street, New York, NY 10032, USA
}

Submitted 27 October 2010: Accepted 12 January 2011: First published online 18 March 2011

\begin{abstract}
Objective: To identify predominant dietary patterns among Hispanic women and to determine whether adherence to dietary patterns is predicted by neighbourhoodlevel factors: linguistic isolation, poverty rate and the retail food environment.

Design: Cross-sectional analyses of predictors of adherence to dietary patterns identified from principal component analysis of data collected using the Study of Women's Health Across the Nation FFQ. Census data were used to measure poverty rates and the percentage of Spanish-speaking families in the neighbourhood in which no person aged $\geq 14$ years spoke English very well (linguistic isolation) and the retail food environment was measured using business listings data.

Setting: New York City.

Subjects: A total of 345 Hispanic women.

Results: Two major dietary patterns were identified: a healthy dietary pattern loading high for vegetables, legumes, potatoes, fish and other seafood, which explained 17\% of the variance in the FFQ data and an energy-dense dietary pattern loading high for red meat, poultry, pizza, french fries and high-energy drinks, which explained $9 \%$ of the variance in the FFQ data. Adherence to the healthy dietary pattern was positively associated with neighbourhood linguistic isolation and negatively associated with neighbourhood poverty. Presence of more fast-food restaurants per square kilometre in the neighbourhood was significantly associated with lower adherence to the healthy diet. Adherence to the energy-dense dietary pattern was inversely, but not significantly, associated with neighbourhood linguistic isolation.

Conclusions: These results are consistent with the hypothesis that living in immigrant enclaves is associated with healthy dietary patterns among Hispanics.
\end{abstract}

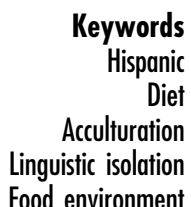

eywords

ispanic

Diet

Linguistic isolation
Racial and ethnic minority populations have been acutely affected by the epidemic of overweight and obesity in the USA. Hispanics, along with Black Americans and Native Americans, face a higher risk of obesity than Caucasians $^{(1)}$. Multiple studies have shown that firstgeneration immigrants have a smaller body size compared with second- and third-generation immigrants and that the number of years since immigration is correlated with increased body size ${ }^{(2-7)}$. These studies have been interpreted to suggest that, over time, immigrants adapt to the 'obesogenic' environment of the USA, which promotes dietary and physical activity patterns favouring a positive energy balance, and thus gain weight ${ }^{(3-6)}$. Thus, acculturation, conceptualized as the process by which immigrants adopt the cultural norms of the host society (in this case dietary and physical activity patterns), has been identified as an important topic for studying body size among Hispanics ${ }^{(4,8,9)}$.

There is a growing volume of literature showing that, among Hispanics, increasing generation since immigration, duration of residence in the USA and other measures of increased acculturation are associated with diets that are lower in rice, beans and fruit and higher in sugar and sugar-sweetened drinks ${ }^{(10)}$. Much of the research has focused on individual-level measures of acculturation, such as the respondent's use of English or years of residence in the USA. However, a few studies have shown that residence in primarily immigrant and co-ethnic neighbourhoods, areas likely to have groceries and restaurants selling familiar home country foods ${ }^{(11)}$, is linked to healthier diets ${ }^{(12-14)}$. Although presented as an indicator of neighbourhood-level acculturation status, foreign-born 
composition is, conceptually, a less direct measure of neighbourhood-level immigrant acculturation than are measures of linguistic isolation ${ }^{(7)}$. Linguistic isolation is a term used by the census to characterize households in which no person aged $\geq 14$ years speaks English 'very well' (http://www.doleta.gov/reports/CensusData/Glossary.cfm). For example, neighbourhoods could have similar proportions of immigrant residents, but the neighbourhoods could differ in levels of linguistic isolation and thus the extent to which they are isolated from obesogenic dietary norms and practices prevalent in the USA. Neighbourhood linguistic isolation has been found to be a stronger predictor of BMI among Hispanics than neighbourhood foreign-born composition $^{(7)}$. In addition, the association between nativity and BMI was weaker among Hispanics living in neighbourhoods with higher levels of linguistic isolation than among those living in neighbourhoods with lower levels of linguistic isolation ${ }^{(7)}$.

To date, the vast majority of research on immigrant acculturation and diet has focused on consumption of individual or groups of nutrients, such as fats and sugars, or on consumption of food groups, such as fruits and vegetables ${ }^{(10)}$. Referred to as 'nutritionism', this focus on nutrients or food items high in specific nutrients as the predominant outcome of interest has been critiqued as being overly reductionist, failing to account for how foods are eaten in combination as a part of meals and cuisines ${ }^{(15-18)}$. Since nutrients and food items are commonly consumed as part of a dietary pattern or preference, analyses of associations between indicators of acculturation and single nutrients or food items may be confounded by the effects of acculturation on overall dietary preferences. Inter-individual variation in dietary patterns, as opposed to variation in single nutrients, may be a better indicator of the effects of individualand neighbourhood-level acculturation on diet ${ }^{(19)}$.

Using data from FFQ, we sought to identify major dietary patterns among Hispanic women and to evaluate the extent of individual women's adherence to those patterns. We then examined the association of adherence to the dietary patterns with individual- and neighbourhoodlevel acculturation and the neighbourhood retail food environment.

\section{Experimental methods}

\section{Sample}

We analysed sociodemographic and FFQ data for the Hispanic female guardians (typically the mother; alternatively, a grandmother) of children participating in the Head Start Program who were enrolled in a study of risk factors for asthma. The asthma study has been described extensively elsewhere. Head Start, a federal programme that focuses on the healthy development of children between the ages of 3 and 5 years, provides education, health, nutrition and other services to low-income children and their families.
In brief, 1026 children were recruited from fifty Head Start programmes conducted in northern Manhattan, Bronx and Brooklyn; their guardians responded to a questionnaire on asthma symptoms and risk factors ${ }^{(20)}$. Of these, 547 children were enrolled into a substudy involving a home visit during which further questionnaire data were collected and environmental samples were taken ${ }^{(21)}$. Recruitment and data collection occurred between November 2003 and August 2006. The Columbia Presbyterian Medical Center Institutional Review Board approved the study protocol and all participants provided written informed consent.

\section{Individual-level data}

The parents of all the recruited children took part in an extensive questionnaire-based interview and provided sociodemographic information. Data from this questionnaire that were assessed as potential individual-level predictors of diet included Hispanic ethnicity (Mexican, Puerto Rican, Dominican or other/mixed), age, nativity, language used at home, whether the respondent currently attended school, years of schooling completed and whether the respondent worked outside the home. As part of the home visit protocol that the subset of the families participated in, the children's guardians were asked to complete the Block 'Study of Women's Health Across the Nation' (SWAN) FFQ, the Spanish language version of which includes the 103 core SWAN FFQ items, plus nine additional questions on food items common to Hispanic cuisine ${ }^{(22)}$.

\section{Neigbbourbood-level data}

Home addresses were geocoded to the appropriate tax lot using Geosupport software (City of New York, Department of City Planning, New York City, NY, USA), and data on neighbourhood food and social environment characteristics were collected using aerial weighting methods for $0.5 \mathrm{~km}$ radial buffers around each respondent's home. US Census 2000 Summary File 3 data were used to measure neighbourhood sociodemographic characteristics, including: percentage of the population below the federal poverty line, referred to here as 'per cent poverty'; percentage of the population reporting Hispanic ethnicity, referred to here as 'per cent Hispanic'; and percentage of Spanish-speaking households that were linguistically isolated (defined by the Census Bureau as a household in which no person aged $\geq 14$ years speaks English only or speaks a non-English language and speaks English 'very well') and referred to here as 'per cent linguistically isolated'. Data on the locations of supermarkets, grocery stores, produce markets, convenience stores, bodegas, meat stores, fish stores, candy and nut stores, bakeries, fast-food restaurants and pizza parlours in 2005 were obtained from a commercial database purchased from Dun and Bradstreet ${ }^{(23,24)}$. Locations of farmers' markets in 2006 were obtained from New York City Coalition Against Hunger, the Council on the Environment of New York City and the Farmer's Market Federation of New York. 


\section{Statistical analyses}

A modified version of the principal component analysis (PCA) approach described by $\mathrm{Hu}$ et $a l^{(17)}$ for the Health Professionals Follow-up Study was used to identify dietary patterns with the SWAN FFQ data. Hu et al. grouped food items from the Willett FFQ into forty food groups and totalled the servings of each food per day in each group. The food groups used by Hu et al. were used along with the SWAN FFQ with a few modifications. Because the SWAN FFQ includes more soya-based items compared with the Willett FFQ, an additional food group for soya-based products was created. In addition, because participants reported very few servings of alcohol on the FFQ, the separate beer, wine and hard liquor groups used by $\mathrm{Hu}$ et al. were combined into one alcoholic beverage group.

The few previously conducted studies that have used PCA with an FFQ designed for Hispanics provide little guidance on how to group Hispanic food items ${ }^{(25,26)}$. We tried two approaches and compared the dietary patterns they identified. Our first approach was to distribute the Hispanic food items into the food groups devised by $\mathrm{Hu}$ et al. on the basis of culinary usage (e.g. we placed flour tortillas in the refined grains group that includes white breads, muffins and bagels). Our second approach was to place all the Hispanic food items in their own food group. For each approach, we used PCA with orthogonal varimax rotation, retaining components that had eigenvalue $>1$ and were interpretable. A factor score was calculated as a measure of adherence to each component using the observed food group servings and the component loading scores ${ }^{(17)}$.

As in our past studies, because of the large number of types of retail outlets considered, retail food outlets were grouped into categories considered to be 'BMI healthy' (supermarkets, produce markets, farmers markets and health food stores), 'BMI unhealthy' (fast food, pizza, convenience stores, bodegas, meat markets, candy and nut stores) and 'BMI neutral' (restaurants excluding fast food and pizzerias, small grocery stores, fish markets and speciality stores $)^{(23,24)}$. The identification and classification of retail outlets and the rationale for the grouping of outlets have been explained previously in an extensive manner. For the present analyses, we calculate the density (outlets per square kilometre of land area in the $0.5 \mathrm{~km}$ radial buffer) of BMI-healthy, BMI-unhealthy and BMIneutral retail outlets. The density of BMI-healthy food outlets has been found to be inversely associated with maternal obesity and BMI in adults ${ }^{(23,24)}$. In addition, because of the interest among the policy makers of New York City (NYC) in the role of these particular food outlets, separate analyses examined whether the presence or density of fast-food restaurants, pizza parlours, supermarkets and bodegas in the participant's neighbourhood predicted adherence to the dietary patterns.

Generalized estimating equation (GEE) analyses were used to evaluate the association between adherence to the identified dietary patterns and individual- and neighbourhood-level sociodemographic characteristics. Our GEE models used the respondent's community district to designate larger neighbourhood areas in the calculation of robust standard errors ${ }^{(27)}$. Community districts represent named neighbourhoods in NYC such as Central Harlem or Washington Heights in which local community boards have influence over development, zoning and licensing. Analyses began with consideration of the individual-level sociodemographic characteristics; neighbourhood-level variables were subsequently added to the model. The individual-level variables, Hispanic ethnicity, USA or foreign place of birth, and use of English at home, proved to be highly inter-correlated and all three variables could not be entered into models simultaneously. Of the three variables, Hispanic ethnicity and language used at home proved to be the least inter-correlated, and analyses focused on these variables. Neighbourhood per cent Hispanic and per cent linguistically isolated were strongly correlated $(r=0.55)$; because linguistic isolation had greater face validity as a measure of acculturation status, analyses concentrated on this variable as a measure of neighbourhood-level acculturation status. Since neighbourhood-level socio-economic status may have an effect on diet above and beyond being just a proxy of individual-level socio-economic status and may also correlate with neighbourhood immigrant composition, we included neighbourhood per cent poverty in the model as a potential confounder. The neighbourhood retail food environment variables were added to the statistical model to evaluate whether differences in the density of retail outlets explained differences in diet by individual- and neighbourhood-level sociodemographic characteristics.

\section{Results}

From the 547 families who took part in the home visit portion of the study, a total of 355 female guardians, who self-reported their ethnicity as Hispanic, provided FFQ data. Of them, 345 provided the complete sociodemographic data required for the present analyses. Table 1 provides descriptive statistics for the study population.

The PCA of the FFQ data with Hispanic food items distributed across food groups identified two major components. The first component loaded high on vegetables, legumes, potatoes and fish and seafood and explained $17 \cdot 28 \%$ of the variance in the data. This component was named the 'Healthy diet'. The second component loaded high on processed meat, high-energy drinks, fries, poultry, pizza and red meat and explained $9 \cdot 15 \%$ of the variance in the data. This component was named the 'Energy-dense diet'. Table 2 shows the top ten loading items for the healthy diet and the energy-dense diet. The two approaches used to group the Hispanic food items in the FFQ produced almost identical results, 
a $99 \%$ correlation for adherence to the healthy diet and a 94\% correlation for adherence to the energy-dense diet. We therefore focused on the results from the PCA based on distributing the Hispanic food items among the existing food groups.

Table 3 shows the results of GEE analyses of predictors of adherence to the healthy dietary pattern. Model 1 assessed associations between individual-level sociodemographic characteristics and adherence to the healthy dietary pattern. Compared with those reporting a Mexican ethnicity, those reporting a Puerto Rican ethnicity had lower adherence to the healthy dietary pattern. Adherence was also associated with being in school, increasing age and total estimated energy consumption, but was not associated with use of English at home. Model 2 included variables for individual and neighbourhood sociodemographic characteristics. Adherence to the healthy dietary pattern was significantly negatively associated with increasing neighbourhood poverty and significantly positively associated with linguistic isolation. Model 3 included all sociodemographic characteristics as well as characteristics of the neighbourhood

Table 1 Descriptive statistics of the full study population

\begin{tabular}{lcc}
\hline & \multicolumn{2}{c}{ Sample size $(n$ 345) } \\
\cline { 2 - 3 } Categorical individual-level variables & $n$ & $\%$ \\
\hline Hispanic ethnicity & & \\
$\quad$ Mexican & 141 & 41 \\
$\quad$ Dominican & 97 & 28 \\
$\quad$ Puerto Rican & 41 & 12 \\
$\quad$ Other Hispanic & 66 & 19 \\
Current schooling & 261 & 76 \\
$\quad$ Not in school & 84 & 24 \\
$\quad$ In school & 261 & 76 \\
Work status & 84 & 24 \\
$\quad$ Not employed & & \\
$\quad$ Employed & 83 & 24 \\
Language used at home & 262 & 76 \\
$\quad$ English & & \\
$\quad$ Spanish & Mean & Median \\
\cline { 2 - 3 } & & \\
Continuous individual-level variables & $10 \cdot 45$ & $12 \cdot 00$ \\
$\quad$ Years of education & $32 \cdot 35$ & $31 \cdot 22$ \\
$\quad$ Age in years & & \\
\hline
\end{tabular}

food environment. As the densities of the three types of food outlets (BMI healthy, unhealthy and neutral) are strongly correlated (Spearman's rank correlations between 0.60 and 0.82 ), initial analyses considered each of the three types of food outlets as predictors of adherence to the healthy dietary pattern separately and then a single model was fitted to consider all three types of outlets concurrently. The results were consistent regardless of modelling approach: the densities of BMI-healthy, BMI-unhealthy and BMI-neutral retail food outlets were not associated with adherence to the healthy dietary pattern, and inclusion of these variables in the model did not alter the associations between neighbourhood sociodemographic characteristics and adherence. In separate analyses (data not shown) considering fast-food restaurants, pizzerias, supermarkets and bodegas, the only association observed for these specific food outlets was an inverse association between the density of fast-food restaurants and adherence to the healthy diet $(\beta=-0 \cdot 03, P=0 \cdot 02)$. In these analyses, poverty rate and the proportion of Spanish-speaking households that were linguistically isolated remained significant predictors of adherence to the healthy diet.

Table 4 shows the results of GEE analyses of predictors of adherence to the energy-dense dietary pattern. Results for model 1 show that, compared with respondents who reported Mexican ethnicity, those reporting Puerto Rican ethnicity were more adherent to the energy-dense dietary pattern. Adherence was also associated with employment outside the home and with total energy consumption and inversely associated with increasing age. Model 2 showed that adherence to the energy-dense dietary pattern was significantly positively associated with increasing neighbourhood poverty. Model 3 includes additional variables for the characteristics of the neighbourhood food environment. Of the food environment variables, adherence to the energy-dense dietary pattern was positively associated with the density of BMI-neutral retail outlets. A large component of the BMI-neutral retail outlet category consists of restaurants other than fast food or pizzerias, and the association between adherence to the energydense dietary pattern and the density of BMI-neutral retail outlets was driven almost entirely by the association

Table 2 Principal components identified in the FFQ data

\begin{tabular}{lcllc}
\hline \multicolumn{2}{c}{ Component 1: Healthy diet } & & \multicolumn{2}{c}{ Component 2: Energy-dense diet } \\
\cline { 1 - 2 } Food group & Loading weight & & Food group & Loading weight \\
\hline Cruciferous vegetables & 0.75 & & Processed meat & 0.67 \\
Green leafy vegetables & 0.74 & & High-energy drinks & 0.61 \\
Dark-yellow vegetables & 0.68 & & Fries & 0.58 \\
Legumes & 0.62 & Poultry & 0.50 \\
Potatoes & 0.55 & Pizza & 0.44 \\
Fish and other seafood & 0.52 & Red meat & 0.39 \\
Fruit & 0.51 & & Eggs & 0.38 \\
Other vegetables & 0.38 & & Fish and other seafood & 0.35 \\
Soup & 0.32 & Potatoes & 0.29 \\
\hline
\end{tabular}


Table 3 Predictors of adherence to the healthy dietary pattern

\begin{tabular}{|c|c|c|c|c|c|c|}
\hline \multirow[b]{2}{*}{ Predictor } & \multicolumn{2}{|c|}{ Model 1} & \multicolumn{2}{|c|}{ Model 2} & \multicolumn{2}{|c|}{ Model 3} \\
\hline & $\beta$ coefficient & $P$ value & $\beta$ coefficient & $P$ value & $\beta$ coefficient & $P$ value \\
\hline \multicolumn{7}{|l|}{ Hispanic ethnicity } \\
\hline Mexican & Ref. & & Ref. & & Ref. & \\
\hline Dominican & 0.23 & $0 \cdot 13$ & $0 \cdot 25$ & $0 \cdot 12$ & 0.24 & $0 \cdot 13$ \\
\hline Puerto Rican & -0.56 & 0.02 & -0.44 & 0.08 & -0.44 & 0.08 \\
\hline Others & -0.06 & 0.68 & -0.03 & 0.79 & -0.02 & 0.87 \\
\hline Currently in school & 0.28 & 0.02 & 0.23 & 0.08 & $0 \cdot 24$ & 0.06 \\
\hline Currently employed & -0.07 & 0.59 & -0.06 & 0.62 & -0.07 & 0.60 \\
\hline English used at home & 0.27 & $0 \cdot 19$ & $0 \cdot 27$ & $0 \cdot 18$ & $0 \cdot 26$ & 0.19 \\
\hline Age in years & 0.02 & 0.04 & 0.02 & 0.05 & 0.02 & 0.06 \\
\hline Years of education & 0.02 & $0 \cdot 21$ & 0.02 & $0 \cdot 14$ & 0.02 & $0 \cdot 14$ \\
\hline Total energy intake (per $100 \mathrm{kcal})^{*}$ & 0.02 & 0.00 & 0.02 & 0.00 & 0.02 & 0.00 \\
\hline Neighbourhood poverty rate & & & $-1 \cdot 93$ & 0.00 & $-2 \cdot 26$ & 0.00 \\
\hline Neighbourhood per cent Spanish language linguistic isolation & & & $2 \cdot 72$ & 0.00 & $2 \cdot 66$ & 0.00 \\
\hline Density of healthy food outlets (outlets $/ \mathrm{km}^{2}$ ) & & & & & 0.01 & 0.61 \\
\hline Density of unhealthy food outlets (outlets $/ \mathrm{km}^{2}$ ) & & & & & 0.00 & $0 \cdot 27$ \\
\hline Density of neutral food outlets (outlets $/ \mathrm{km}^{2}$ ) & & & & & 0.00 & $0 \cdot 29$ \\
\hline
\end{tabular}

Ref., reference category.

${ }^{*} 1 \mathrm{kcal}=4 \cdot 184 \mathrm{~kJ}$.

Table 4 Predictors of adherence to the energy-dense dietary pattern

\begin{tabular}{|c|c|c|c|c|c|c|}
\hline \multirow[b]{2}{*}{ Predictor } & \multicolumn{2}{|c|}{ Model 1} & \multicolumn{2}{|c|}{ Model 2} & \multicolumn{2}{|c|}{ Model 3} \\
\hline & $\beta$ coefficient & $P$ value & $\beta$ coefficient & $P$ value & $\beta$ coefficient & $P$ value \\
\hline \multicolumn{7}{|l|}{ Hispanic ethnicity } \\
\hline Mexican & Ref. & & Ref. & & Ref. & \\
\hline Dominican & $0 \cdot 12$ & $0 \cdot 19$ & $0 \cdot 12$ & $0 \cdot 17$ & $0 \cdot 15$ & $0 \cdot 11$ \\
\hline Puerto Rican & $0 \cdot 74$ & 0.00 & $0 \cdot 71$ & 0.00 & $0 \cdot 71$ & 0.00 \\
\hline Others & $-0 \cdot 12$ & $0 \cdot 19$ & -0.09 & $0 \cdot 26$ & $-0 \cdot 12$ & $0 \cdot 12$ \\
\hline Currently in school & -0.12 & $0 \cdot 19$ & -0.09 & 0.26 & -0.12 & $0 \cdot 12$ \\
\hline Currently employed & $0 \cdot 17$ & 0.05 & 0.17 & 0.05 & 0.18 & 0.06 \\
\hline English used at home & $0 \cdot 11$ & $0 \cdot 27$ & $0 \cdot 12$ & $0 \cdot 24$ & $0 \cdot 13$ & $0 \cdot 15$ \\
\hline Age in years & -0.02 & 0.00 & -0.02 & 0.00 & -0.02 & 0.00 \\
\hline Years of education & -0.01 & $0 \cdot 29$ & -0.01 & $0 \cdot 35$ & -0.01 & 0.43 \\
\hline Total energy intake (per $100 \mathrm{kcal})^{\star}$ & 0.04 & 0.00 & 0.04 & 0.00 & 0.04 & 0.00 \\
\hline Neighbourhood poverty rate & & & 1.05 & 0.01 & $2 \cdot 06$ & 0.00 \\
\hline Neighbourhood per cent Spanish language linguistic isolation & & & -0.38 & 0.53 & $-1 \cdot 83$ & 0.05 \\
\hline Density of healthy food outlets (outlets $/ \mathrm{km}^{2}$ ) & & & & & 0.00 & 0.77 \\
\hline Density of unhealthy food outlets (outlets $/ \mathrm{km}^{2}$ ) & & & & & 0.00 & 0.32 \\
\hline Density of neutral food outlets (outlets $/ \mathrm{km}^{2}$ ) & & & & & 0.01 & 0.03 \\
\hline
\end{tabular}

Ref., reference category.

${ }^{*} 1 \mathrm{kcal}=4 \cdot 184 \mathrm{~kJ}$.

between adherence to the energy-dense dietary pattern and the density of restaurants other than fast food or pizzerias $\left(\beta=0.006\right.$ per restaurant $\left./ \mathrm{km}^{2}, P=0.03\right)$. Density of other types of specific retail food outlets was not associated with adherence to the energy-dense dietary pattern. A comparison between models 2 and 3 shows that the inverse association between linguistic isolation and adherence to the energy-dense dietary pattern increases and reaches borderline statistical significance when the food environment variables are included in analyses.

\section{Discussion}

In the present population of low-income Hispanic women, two major dietary patterns were identified: one that could be construed as being healthy, high in vegetables, fruit and legumes (Table 2); and a second that could be construed as being less healthy, comprised of energydense foods (Table 2). In our study, adherence to the healthy diet was strongly predicted by neighbourhood linguistic isolation, even after control for neighbourhood poverty. The association with neighbourhood linguistic isolation could not be accounted for by differences in the neighbourhood retail food environment. Adherence to the energy-dense dietary pattern was initially modestly inversely, but not significantly, associated with neighbourhood linguistic isolation, an association that increased after adjustment for measures of the neighbourhood retail food environment.

These results are consistent with the hypothesis that living in immigrant enclaves is associated with healthy 
dietary patterns among Hispanic immigrants ${ }^{(12,14)}$. Past research among Hispanics has found associations between measures of acculturation and diet, but this work has focused mainly on specific nutrients or consumption of specific food groups such as fruits and vegetables, and individual-level measures of acculturation such as the participant's use of English. However, nutrients and food items are not consumed in isolation; analyses that focus on isolated nutrients or food groups as the measure of diet may find associations between indicators of acculturation and diet that are not observed when the overall dietary pattern is considered as the outcome of interest ${ }^{(17)}$. For instance, past studies among immigrants have found associations between speaking English and the consumption of food items, but we did not observe an association between the participant's use of English and overall dietary pattern ${ }^{(10)}$. In our analysis, neighbourhood-level acculturation is a major correlate of dietary patterns, appearing to promote one pattern of consumption while protecting a second pattern.

The nine additional food items included in the SWAN FFQ to reflect Hispanic cuisine did not, as a group, load highly on either the identified healthy or energy-dense dietary patterns. Since these 'Hispanic' FFQ items include not only foods that could be considered healthy (e.g. cassava or cooked green peppers) but also foods that could be considered energy dense (e.g. condensed milk or flan), the failure of this grouping to load highly on either pattern is not surprising. Thus, neighbourhood linguistic isolation does not appear to promote consumption of the 'Hispanic' food items added to the 103-item core SWAN FFQ but rather the consumption of an overall dietary pattern high in vegetables, legumes, fish, seafood and fruit, items that are part of the core FFQ. If, as literature suggests, the rise in obesity among immigrants results, at least in part, from the loss of home country diets and acquisition of obesogenic American diets, interventions that facilitate the maintenance of home country diets may make important contributions to obesity prevention $^{(10,28,29)}$. Our results suggest, however, that such interventions should focus on the maintenance of patterns of home country dietary practices that are healthy (e.g. patterns that maintain higher levels of consumption of fruit, rice and legumes and lower sugar consumption) rather than on the consumption of specific food items or even types of cuisines considered to be 'traditional' ${ }^{\text {,(10) }}$. Lee et $a l^{(30)}$ suggest that, in the face of increasing market penetration of processed foods and US chain restaurants in Korea, a multi-pronged national campaign to promote retention of the vegetable-centred traditional Korean diet resulted in significant positive health effects. A similar approach that acknowledges the healthy dietary patterns and their practices that many immigrants bring from their countries of origin, and encouragement of the retention of the best elements of those practices, an approach Yeh et al. refer to as 'selective acculturation'(29,31,32), may be of use among immigrant groups in the USA.
In these analyses, our construct of 'BMI-healthy' retail outlets, which has previously been shown to predict lower BMI in adults and lower risk of maternal obesity ${ }^{(23,24)}$, did not predict adherence to either of the identified dietary patterns. Whereas adherence to the healthy dietary pattern might be expected to be associated with lower energy intake and the energy-dense dietary pattern to be associated with higher energy intake, higher energy intake was positively associated with higher adherence to both dietary patterns. Since our previous research has found an association between the density of BMI-healthy outlets and lower BMI, we considered that control for energy intake in models that include food environment variables might represent over-adjustment for a potential intervening variable. However, the results for the food environment variables were not materially different in analyses that did not control for energy intake. The only association between our previously defined food environment measures and diet was between a higher density of BMIneutral outlets and higher adherence to the energy-dense dietary pattern, an association primarily driven by the density of restaurants other than fast food or pizza. It is likely that an abundance of restaurants is linked to higher consumption of restaurant meals and exposure to nonhome country cuisines and higher levels of acculturation. In addition, meals consumed outside the home are less healthy than meals prepared at home ${ }^{(33,34)}$. In the analyses examining specific retail outlets, the only association observed was between higher density of fast-food restaurants and lower adherence to the healthy dietary pattern. Although prior work has not found strong links between the presence of fast-food outlets and diet or obesity, it is possible that in this population an abundance of fast-food restaurants is linked to more meals being consumed in these restaurants ${ }^{(24,35-37)}$. Such restaurants are not major sources of the vegetables, legumes and fruit associated with the healthy dietary pattern. Although there were a few associations between the dietary pattern data and aspects of the retail food environment, the food environment measures did not explain the association between neighbourhood Spanish language linguistic isolation and adherence to the healthy dietary pattern.

In conclusion, these analyses suggest that measures of neighbourhood-level immigrant acculturation may predict differences in dietary patterns among Hispanic women that cannot be explained by variation in the neighbourhood retail food environment. The present study contributes to a small but growing literature that considers how neighbourhood contexts may moderate the influence of individual ethnicity or acculturation on health behaviour. Strengths of the study include the representation of several Hispanic ethnicities in the study sample, the use of objective measures of the food environment and the use of dietary pattern analysis to characterize the food intake of participants. The primary limitation is the use of a sample that includes only women. Interventions and nutritional 
counselling that focus on preserving and promoting the home country diets of immigrants may be effective in preventing the weight gain that is commonly seen among immigrants with longer duration of residence in the USA and among successive generations of immigrants.

\section{Acknowledgements}

The research was funded by grants from the National Institutes of Health (R01ES014229 and R01HL068236). The authors have no conflict of interest to declare. Y.P. conceptualized the research questions, directed the analyses and drafted the manuscript; K.N. helped design the neighbourhood health effects study and co-authored the manuscript; J.Q. developed the Geographic Information System measures of neighbourhood characteristics; C.W. helped design the neighbourhood health effects study, developed the statistical analytical plan and co-authored the manuscript; J.J. designed the asthma study and oversaw participant recruitment/data collection; A.R. designed the neighbourhood effects study, conducted the statistical analyses and co-authored the manuscript.

\section{References}

1. Flegal KM, Carroll MD, Ogden CL et al. (2010) Prevalence and trends in obesity among US adults, 1999-2008. JAMA 303, 235-241.

2. Popkin BM \& Udry JR (1998) Adolescent obesity increases significantly in second and third generation US immigrants: the National Longitudinal Study of Adolescent Health. J Nutr 128, 701-706.

3. Kaplan MS, Huguet N, Newsom JT et al. (2004) The association between length of residence and obesity among Hispanic immigrants. Am J Prev Med 27, 323-326.

4. Gordon-Larsen P, Mullan Harris K, Ward DS et al. (2003) Acculturation and overweight-related behaviors among Hispanic Immigrants to the US: the National Longitudial Study of Adolescent Health. Soc Sci Med 57, 2023-2034.

5. Goel MS, McCarthy EP, Phillips RS et al. (2004) Obesity among US immigrant subgroups by duration of residence. JAMA 292, 2860-2867.

6. Lauderdale DS \& Rathouz PJ (2000) Body mass index in a US national sample of Asian Americans: effects of nativity, years since immigration and socioeconomic status. Int $J$ Obes Relat Metab Disord 24, 1188-1194.

7. Park Y, Neckerman KM, Quinn J et al. (2008) Significance of place of birth and place of residence and their relationship to BMI among immigrant groups in New York City. Int J Behav Nutr Phys Act 5, 1-35.

8. Sundquist J \& Winkleby M (2000) Country of birth, acculturation status and abdominal obesity in a national sample of Mexican-American women and men. Int $J$ Epidemiol 29, 470-477.

9. Himmelgreen DA, Perez-Escamilla R, Martinez D et al. (2004) The longer you stay, the bigger you get: length of time and language use in the US are associated with obesity in Puerto Rican women. Am J Phys Anthropol 125, 90-96.

10. Ayala GX, Baquero B \& Klinger S (2008) A systematic review of the relationship between acculturation and diet among Latinos in the United States: implications for future research. J Am Diet Assoc 108, 1330-1344.
11. Lee DO (1995) Korea town and Korean small firms in Los-Angeles - locating in the ethnic neighborhoods. Prof Geogr 47, 184-195.

12. Osypuk TL, Diez Roux AV, Hadley C et al. (2009) Are immigrant enclaves healthy places to live? The Multi-Ethnic Study of Atherosclerosis. Soc Sci Med 69, 110-120.

13. Reyes-Ortiz CA, Ju H, Eschbach K et al. (2009) Neighbourhood ethnic composition and diet among MexicanAmericans. Public Health Nutr 12, 2293-2301.

14. Dubowitz T, Subramanian SV, Acevedo-Garcia D et al. (2008) Individual and neighborhood differences in diet among low-income foreign and US-born women. Womens Health Issues 18, 181-190.

15. Scrinis G (2008) On the ideology of nutritionism. Gastronomica 8, 39-48.

16. Dixon J (2009) From the imperial to the empty calorie: how nutrition relations underpin food regime transitions. Agric Human Values 26, 321-333.

17. Hu FB, Rimm E, Smith-Warner SA et al. (1999) Reproducibility and validity of dietary patterns assessed with a food-frequency questionnaire. Am J Clin Nutr 69, 243-249.

18. Hu FB (2002) Dietary pattern analysis: a new direction in nutritional epidemiology. Curr Opin Lipidol 13, 3-9.

19. Lin H, Bermudez OI \& Tucker KL (2003) Dietary patterns of Hispanic elders are associated with acculturation and obesity. J Nutr 133, 3651-3657.

20. Jacobson JS, Goldstein IF, Canfield SM et al. (2008) Early respiratory infections and asthma among New York City Head Start children. $J$ Asthma 45, 301-308.

21. Rundle A, Goldstein IF, Mellins RB et al. (2009) Physical activity and asthma symptoms among New York City Head Start Children. J Asthma 46, 803-809.

22. Block G, Wakimoto P, Jensen C et al. (2006) Validation of a food frequency questionnaire for Hispanics. Prev Chronic Dis 3, A77.

23. Janevic T, Borrell L, Savitz D et al. (2010) Neighborhood food environment and gestational diabetes in New York City. Paediatr Perinat Epidemiol 24, 249-254.

24. Rundle A, Neckerman KM, Freeman L et al. (2009) Neighborhood food environment and walkability predict obesity in New York City. Environ Health Perspect 117, 442-447.

25. Nettleton JA, Steffen LM, Mayer-Davis EJ et al. (2006) Dietary patterns are associated with biochemical markers of inflammation and endothelial activation in the MultiEthnic Study of Atherosclerosis (MESA). Am J Clin Nutr 83, 1369-1379.

26. Tseng M, DeVellis RF, Maurer KR et al. (2000) Food intake patterns and gallbladder disease in Mexican Americans. Public Health Nutr 3, 233-243.

27. Lovasi GS, Quinn JW, Rauh VA et al. (2011) Chlorpyrifos exposure and urban residential environment characteristics as determinants of early childhood neurodevelopment. Am J Public Health 101, 63-70.

28. Pham KL, Harrison GG \& Kagawa-Singer M (2007) Perceptions of diet and physical activity among California Hmong adults and youths. Prev Chronic Dis 4, A93.

29. Harrison GG, Kagawa-Singer M, Foerster SB et al. (2005) Seizing the moment: California's opportunity to prevent nutrition-related health disparities in low-income Asian American populations. Cancer 15, 2962-2968.

30. Lee M-J, Popkin BM \& Kim S (2002) The unique aspects of the nutrition transition in South Korea: the retention of healthful elements in their traditional diet. Public Health Nutr 5, 197-203.

31. McArthur LH, Anguiano RPV \& Nocetti D (2001) Maintenance and change in the diet of Hispanic immigrants in Eastern North Carolina. Fam Consum Sci Res J 29, 309-335. 
32. Yeh MC, Viladrich A, Bruning N et al. (2009) Determinants of Latina obesity in the United States: the role of selective acculturation. J Transcult Nurs 20, 105-115.

33. Guthrie JF, Lin BH \& Frazao E (2002) Role of food prepared away from home in the American diet, 1977-78 versus 1994-96: changes and consequences. J Nutr Educ Behav 34, 140-150.

34. Orfanos P, Naska A, Trichopoulos D et al. (2007) Eating out of home and its correlates in 10 European countries. The European Prospective Investigation into Cancer and Nutrition (EPIC) study. Public Health Nutr 10, 1515-1525.
35. Burdette HL \& Whitaker RC (2004) Neighborhood playgrounds, fast food restaurants, and crime: relationships to overweight in low-income preschool children. Prev Med 38, 57-63.

36. Jeffery R, Baxter J, McGuire M et al. (2006) Are fast food restaurants an environmental risk factor for obesity? Int $J$ Behav Nutr Phys Act 3, 2.

37. Liu GC, Wilson JS, Qi R et al. (2007) Green neighborhoods, food retail and childhood overweight: differences by population density. Am J Health Promot 21, 317-325. 